\title{
Updating ambiguous word meanings: a role for the language-selective fronto-temporal network
}

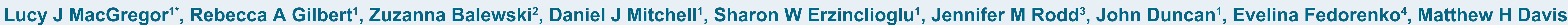
${ }^{1}$ University of Cambridge ${ }^{2}$ University of California at Berkley ${ }^{3}$ University College London ${ }^{4}$ Massachusetts Insitute of Technology

\section{Background}

Word meaning access challenged by semantic ambiguity

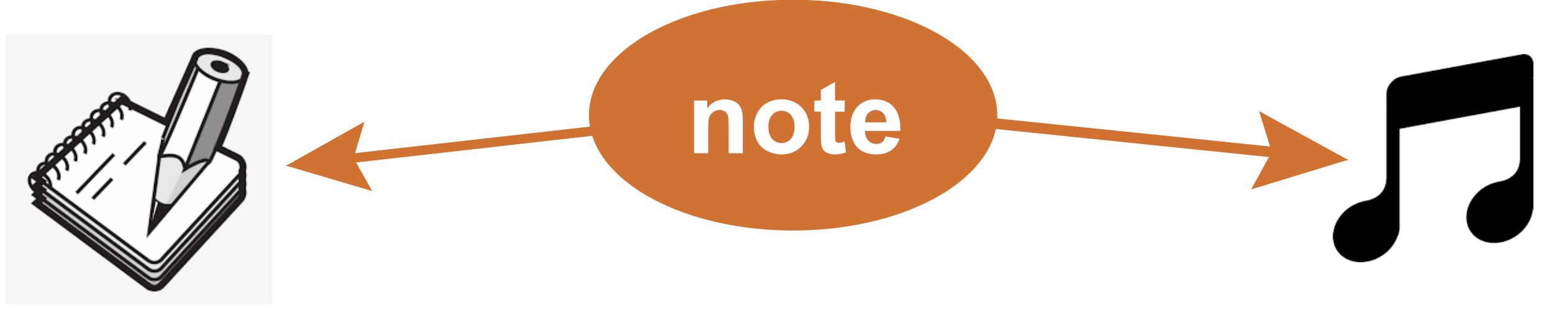

An effective comprehension system can:

* use contextual cues

* adapt in response to recent experience

Is challenging word meaning access supported by:

the domain-specific Language Network ${ }^{[1]}$, and/or the domaingeneral Multiple Demand Network ${ }^{[2]}$ ?

\section{Lesion-Network overlap}

Networks defined from fMRI data in healthy adults ${ }^{[3]}$

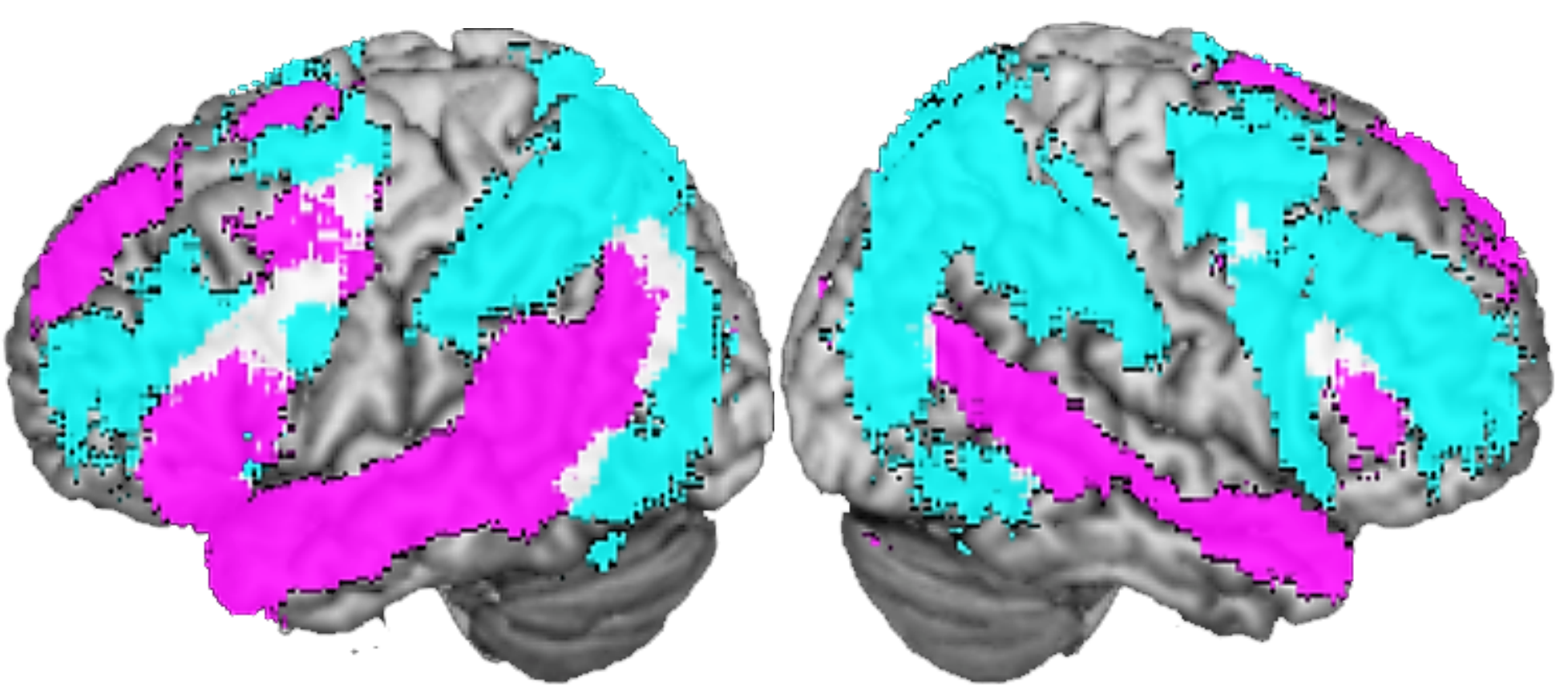

\section{Language Network $(\mathrm{n}=220)$}

MD Network (n=63)

$\square$ Overlap

Probabilistic activation maps thresholded at $5 \%$.

Volunteers $(\mathrm{n}=18)$ with adult-onset chronic cortical lesions recruited from the Cambridge Cognitive Neuroscience Research Panel (CCNRP).
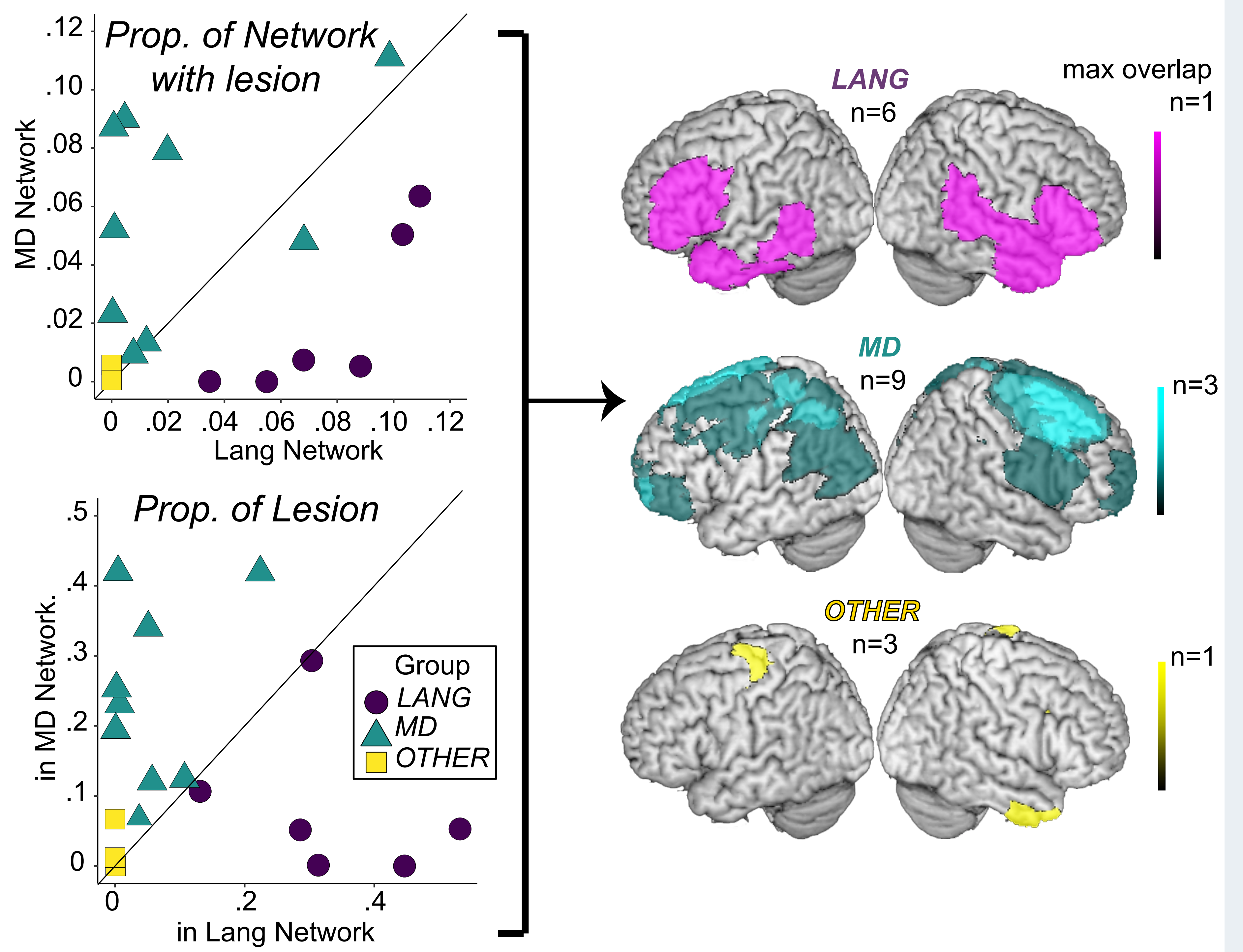

\section{Two-phase experiment}

1. Use of contextual cues during word meaning access Coherence Judgement Task

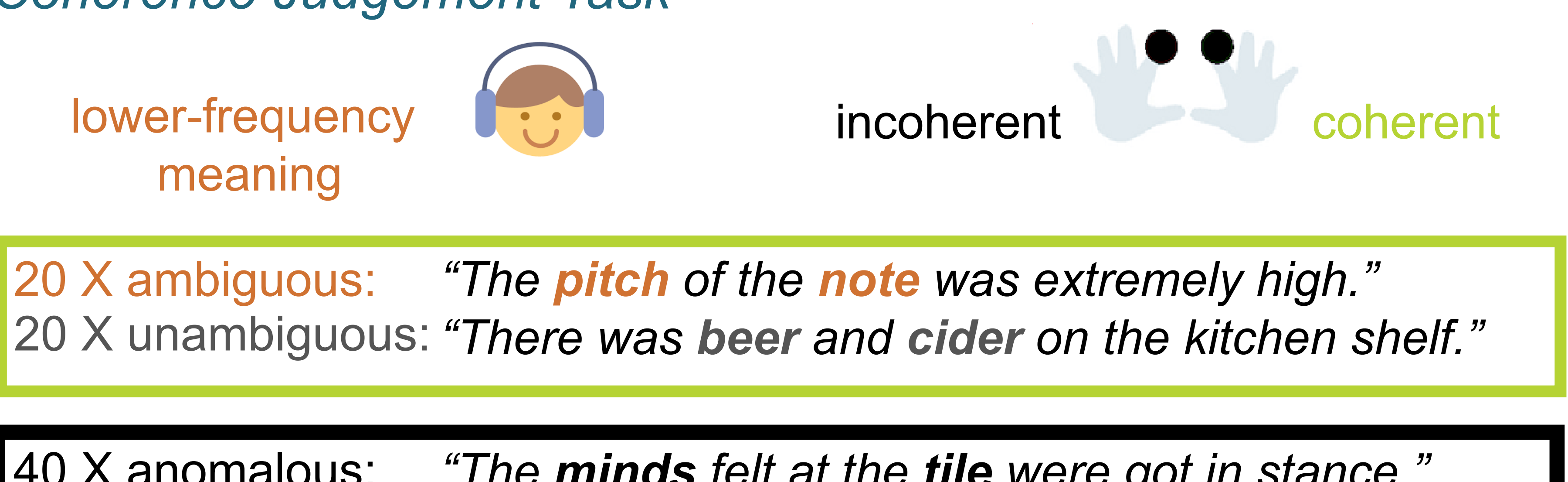

\section{$40 \mathrm{X}$ anomalous: "The minds felt at the tile were got in stance."}

2. Adaptation in response to recent experience

Word Association Task

(counterbalanced
across participants)

\begin{tabular}{|c|c|c|c|}
\hline $40 \mathrm{X}$ primed: & "note" & "note-music" & context consistent \\
\hline & & “note-letter” & $\begin{array}{l}\text { context inconsistent } \\
\text { VS. }\end{array}$ \\
\hline $40 \mathrm{X}$ unprimed: & "star" & $\begin{array}{l}\text { "star-fame" } \\
\text { "star-sky" }\end{array}$ & $\begin{array}{l}\text { context consistent } \\
\text { context inconsistent }\end{array}$ \\
\hline
\end{tabular}

Data analysed with mixed effects models using the 'Ime4' package ${ }^{[4]}$ in R.

\section{Lesion-behaviour assocations}

Individual participant Word Meaning Priming (est. using model residuals):

negatively associated with damage in Language Network, but not MD Network or whole brain.

"not associated with Ambiguity Effect (est. using model residuals) from sentence coherence judgment task.

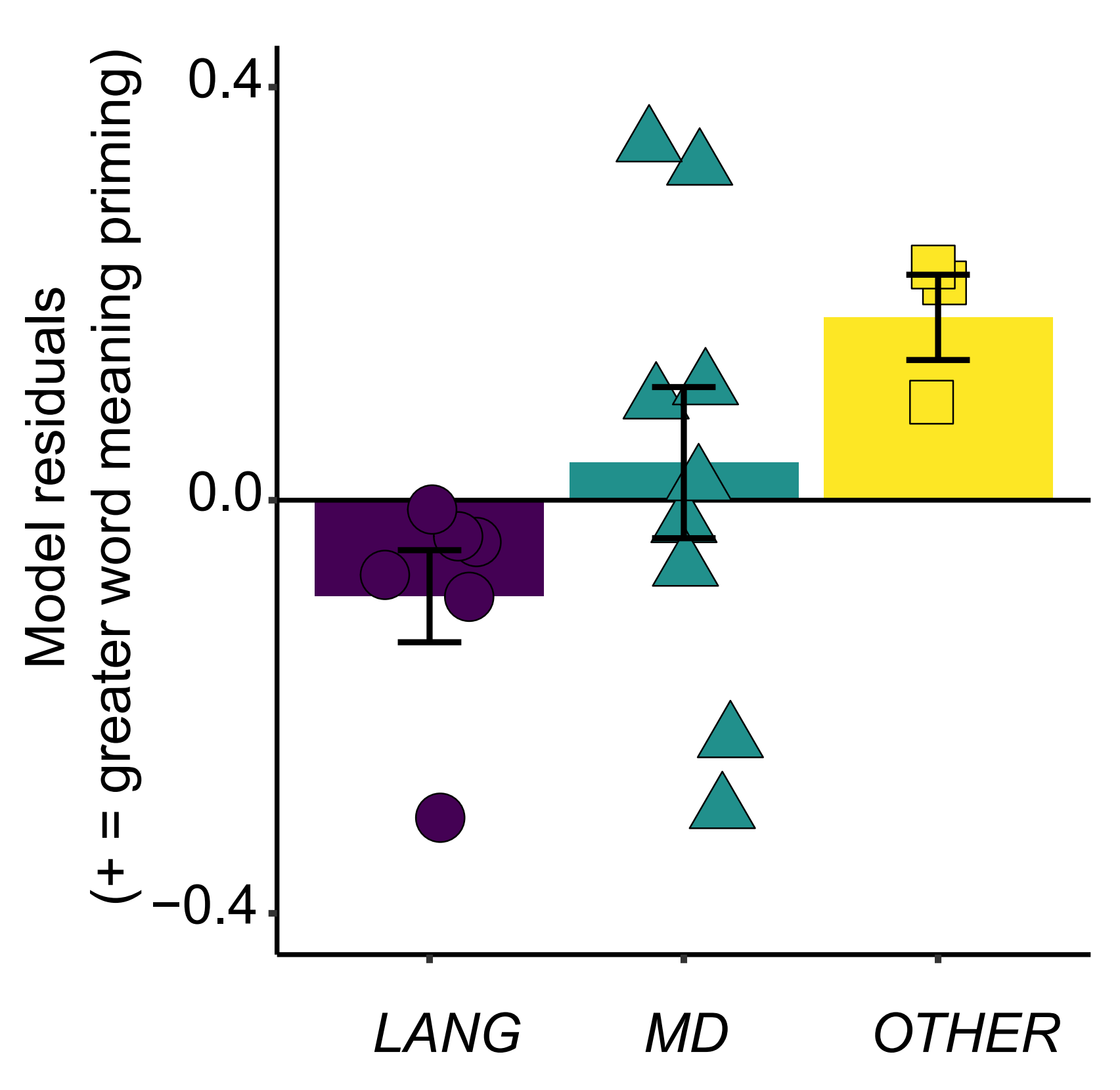

\section{Sentence coherence judgements}

High accuracy $(>90 \%)$ and discriminability (d-prime $>2$ ) for all participants. Response time Ambiguity Effect unaffected by lesion location or extent.
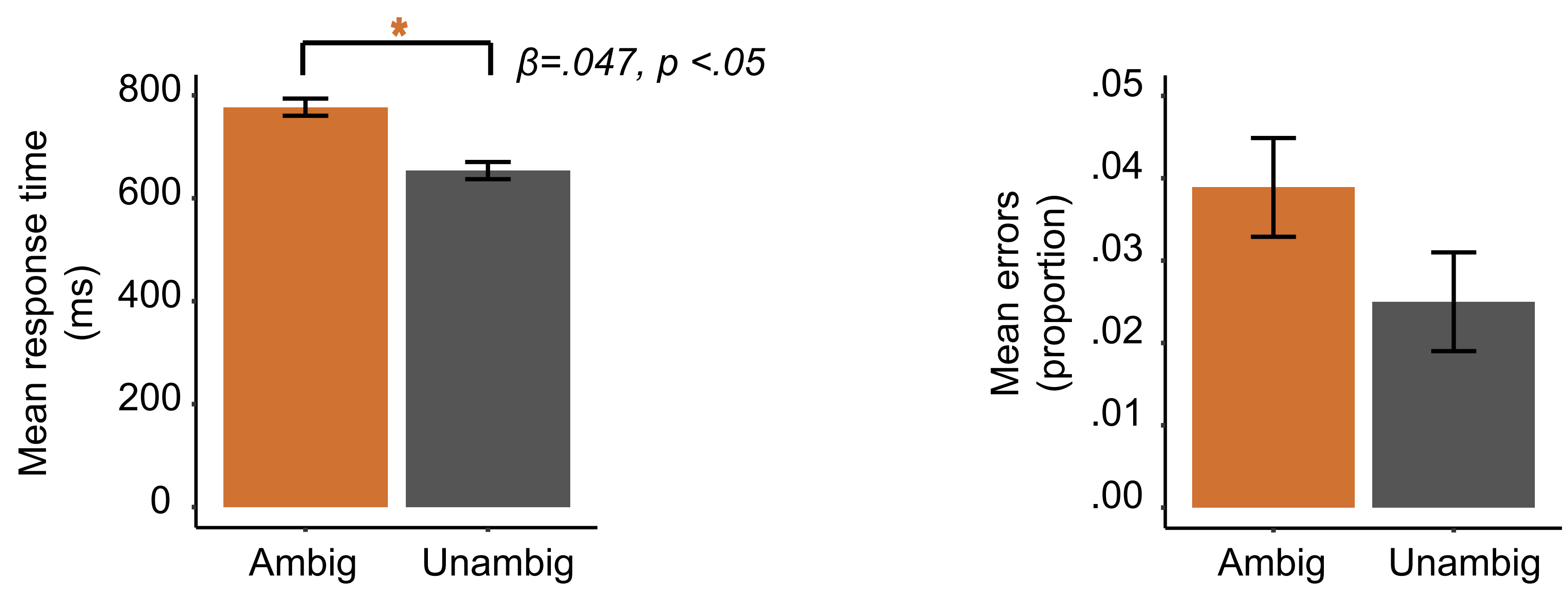

\section{Word asssociation responses}

Word Meaning Priming Effect ${ }^{[5]}$, which did not differ between groups.
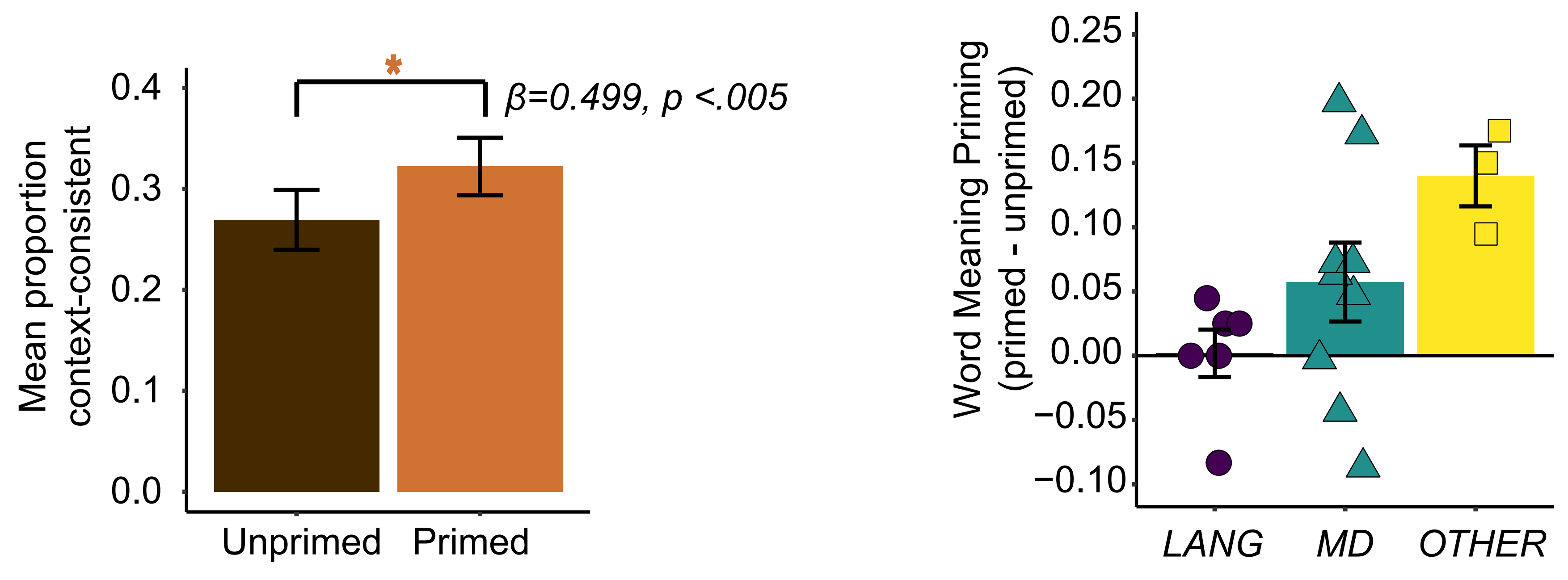

\section{Discussion}

1. Volunteers with lesions to Language and MD Networks. successfully used context to access appropriate lower-frequency ambiguous word meanings during sentence comprehension. 2. Increase in preference for lower frequency word meanings following recent experience (Word Meaning Priming Effect).

3. Extent of adaptation (Word Meaning Priming) was dependent on the Language Network, but not the Multiple Demand Network.

\section{References}

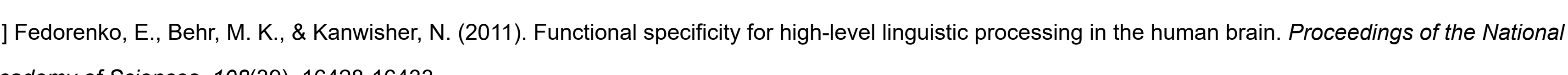

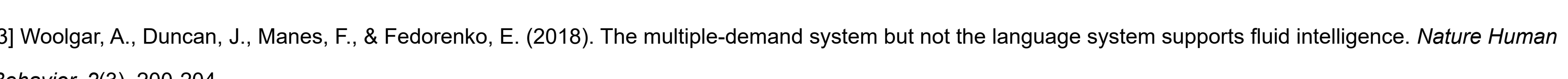
(1)

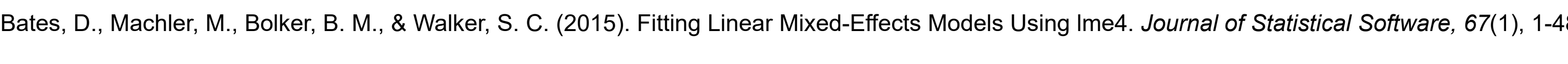

